\title{
Dependence Concepts and Reliability Application of Concomitants of Order Statistics from the Morgenstern Family
}

\author{
Johny Scaria*, Sithara Mohan \\ Department of Statistics, Nirmala College Muvattupuzha, 686661, India
}

\author{
ARTICLE INFO \\ Article History \\ Received 06 Jun 2019 \\ Accepted 01 Jul 2020

\section{Keywords} \\ Concomitants of order statistics \\ Dependence measures \\ Morgenstern type bivariate \\ exponentiated exponential \\ distribution \\ Reliability \\ Series and parallel systems
}

\begin{abstract}
The distribution theory and applications of concomitants from the Morgenstern family of bivariate distributions are discussed in Scaria and Nair, Biom. J. 41 (1999), 483-489. In the present study, some dependence concepts of concomitants of order statistics from the Morgenstern family are discussed. An application in reliability theory of designing a two component system using concomitants is also discussed.
\end{abstract}

(c) 2021 The Authors. Published by Atlantis Press B.V. This is an open access article distributed under the CC BY-NC 4.0 license (http://creativecommons.org/licenses/by-nc/4.0/).

\section{INTRODUCTION}

The concept of concomitants, when the bivariate data are ordered by one of its components, was first introduced by [1]. Let $\left(X_{1}, Y_{1}\right),\left(X_{2}, Y_{2}\right), \ldots,\left(X_{n}, Y_{n}\right)$ be a random sample of size $n$ from a continuous bivariate population with cumulative distribution function (cdf) $F_{X, Y}(x, y)$. If we arrange the $X$-variate in ascending order as $X_{1: n} \leq X_{2: n} \leq \ldots \leq X_{n: n}$, then the $Y$-values paired with these order statistics are denoted by $Y_{[1: n]}, Y_{[2: n]}, \ldots, Y_{[n: n]}$ and are termed as concomitants of order statistics. Recent studies on concomitants of order statistics are in the works of [2-7]. A fine review of works on concomitants of order statistics is available in [8]. Concomitants have found a variety of applications in many applied fields such as selection procedure, ocean engineering, inference problems, prediction analysis and double sampling plans. In particular, ranked set sampling (RSS) proposed by [9] has found a remarkable application of concomitants of order statistics. Inspired from RSS, Scaria and Thomas [10] extended the theory of concomitants and introduced the concept of second order concomitants of order statistics. A noteworthy advantage of employing second order concomitants in sampling is that this technique will reduce the number of measurements required, and therefore reduce the researcher's costs.

Concepts of stochastic dependence is widely discussed in literature and it permeates throughout our daily life. Lai and Xie [11] discussed different dependence concepts, dependence orderings and measures of dependence in detail. The concepts of dependence in the bivariate and multivariate cases are presented in [12,13]. Esary and Proschan [14] gave definitions to the terms right-tail increasing (RTI) and left-tail decreasing (LTD). The term likelihood ratio dependent (LRD) was first defined by [15]. The concept of $T P_{2}$ (totally positive of order 2) was introduced by [16]. For several works of positive dependence we refer to [15,17]. All these concepts are widely used in reliability theory.

Generally, the component life times of a system may be dependent on one another. Hence we need probability models that prescribe the dependence structures among the life time variables. In multi-component systems, measures of dependence between component lives are a major aspect to be considered in selecting the appropriate model. The Morgenstern system of distributions is a popular, and well-known family of bivariate dependent variables, and its numerous generalizations are scattered in the literature. Dependence properties of this family are closely associated with the correlation coefficient although a priori the pivotal parameter of the family is not associated with this concept. It is already established that the Morgenstern family is suitable in reliability modelling [18]. The Morgenstern system of bivariate

*Corresponding author. Email: johny.kakkattil@gmail.com 
distributions discussed in [19] includes all cdfs of the form

$$
F_{X, Y}(x, y)=F_{X}(x) F_{Y}(y)\left[1+\lambda\left\{1-F_{X}(x)\right\}\left\{1-F_{Y}(y)\right\}\right] ;-1 \leq \lambda \leq 1 .
$$

Here the parameter $\lambda$ measures the association between $X$ and $Y$ with spearman's rho $\lambda / 3$ and Kendall's tau 2 $\lambda / 9$. Lai and Xie [11] discussed the dependence structure of the Morgenstern family. No previous work has been done regarding the dependence structure of the concomitants from the Morgenstern family. The present paper is organized as follows. In Section 2, we discuss the dependence concepts of the concomitants from the Morgenstern family. The distribution theory of lifetimes of two component systems using concomitants is discussed in Section 3. In Section 4, we discuss an application of concomitants in reliability modelling of designing a two component system from the Morgenstern type bivariate exponentiated exponential distribution (MTBEED).

\section{CONCOMITANTS OF ORDER STATISTICS AND SOME OF THE DEPENDENCE MEASURES}

The general distribution theory of concomitants from the Morgenstern family is discussed in [20]. The cdf, the probability density function (pdf) of the $r^{\text {th }}$ concomitant $Y_{[r: n]}$ and the joint distribution function of $Y_{[r: n]}$ and $Y_{[s: n]}$ are given in Equations (2.1-2.3), respectively as

$$
\begin{aligned}
& F_{Y_{[r: n]}}(y)=F_{Y}(y)\left[1+\delta_{\lambda_{r}}\left\{1-F_{Y}(y)\right\}\right] ;-1 \leq \lambda \leq 1, \\
& f_{Y_{[r: n]}}(y)=f_{Y}(y)\left[1+\delta_{\lambda_{r}}\left\{1-2 F_{Y}(y)\right\}\right] ;-1 \leq \lambda \leq 1,
\end{aligned}
$$

and

$$
\begin{aligned}
F_{[r, S . n]}\left(y_{1}, y_{2}\right)= & F_{Y}\left(y_{1}\right) F_{Y}\left(y_{2}\right)\left\{1+\delta_{\lambda_{r}}\left[1-F_{Y}\left(y_{1}\right)\right]+\delta_{\lambda_{s}}\left[1-F_{Y}\left(y_{2}\right)\right]\right. \\
& \left.+\delta_{\lambda^{2}}\left[1-F_{Y}\left(y_{1}\right)\right]\left[1-F_{Y}\left(y_{2}\right)\right]\right\} .
\end{aligned}
$$

where $\delta_{\lambda_{r}}=\lambda\left(\frac{n-2 r+1}{n+1}\right), \delta_{\lambda_{s}}=\lambda\left(\frac{n-2 s+1}{n+1}\right)$ and $\delta_{\lambda^{2}}=\lambda^{2}\left\{\frac{n-2 s+1}{n+1}-\frac{2 r(n-2 s)}{(n+1)(n+2)}\right\}$

Equation (2.3) reveals that the joint distribution function of concomitants is a special case of the bivariate Cambanis family, introduced by [21] specified by

$$
\begin{aligned}
F_{X, Y}(x, y)= & F_{X}(x) F_{Y}(y)\left[1+\lambda_{1}\left\{1-F_{X}(x)\right\}+\lambda_{2}\left\{1-F_{Y}(y)\right\}\right. \\
& \left.+\lambda_{3}\left\{1-F_{X}(x)\right\}\left\{1-F_{Y}(y)\right\}\right],
\end{aligned}
$$

with corresponding marginal distributions and joint density function as

$$
\begin{aligned}
& H_{X}(x)=F_{X}(x)\left[1+\lambda_{1}\left\{1-F_{X}(x)\right\}\right], \\
& G_{Y}(y)=F_{Y}(y)\left[1+\lambda_{2}\left\{1-F_{Y}(y)\right\}\right],
\end{aligned}
$$

and

$$
\begin{aligned}
f_{X, Y}(x, y)= & f_{X}(x) f_{Y}(y)\left[1+\lambda_{1}\left\{1-2 F_{X}(x)\right\}+\lambda_{2}\left\{1-2 F_{Y}(y)\right\}\right. \\
& \left.+\lambda_{3}\left\{1-2 F_{X}(x)\right\}\left\{1-2 F_{Y}(y)\right\}\right],
\end{aligned}
$$

When (2.4) is absolutely continuous the parameters satisfy the conditions $\left(1+\lambda_{1}+\lambda_{2}+\lambda_{3}\right)>0,\left(1+\lambda_{1}-\lambda_{2}-\lambda_{3}\right)>0,\left(1-\lambda_{1}+\lambda_{2}-\lambda_{3}\right)>0$ and $\left(1-\lambda_{1}-\lambda_{2}+\lambda_{3}\right)>0$, where $\lambda$ 's are real constants.

Comparing (2.3) with (2.4), we get $\lambda_{1}=\delta_{\lambda_{r}}=\lambda\left(\frac{n-2 r+1}{n+1}\right), \lambda_{2}=\delta_{\lambda_{s}}=\lambda\left(\frac{n-2 s+1}{n+1}\right)$ and $\lambda_{3}=\delta_{\lambda^{2}}=$ $\lambda^{2}\left\{\frac{n-2 s+1}{n+1}-\frac{2 r(n-2 s)}{(n+1)(n+2)}\right\}$.

Concepts of stochastic dependence are widely applicable in statistics. Among the dependence concepts, correlation is still the most widely used concept in applications. Some of the positive dependence measures are discussed in [11]. In this work we deal with the association measures (Kendall's tau and Spearman's rho) of concomitants of order statistics from the bivariate Morgenstern family. 
Holland and Wang [22,23], defined a local dependence function

$$
\gamma(x, y)=\frac{\partial^{2} \log f(x, y)}{\partial x \partial y}
$$

assuming the partial derivative of the second order exists and $f(x, y)$ is the pdf of the bivariate distribution. Holland and Wang [23] also showed that $\gamma(x, y) \geq 0$ (for all $x$ and for all $y$ ) is equivalent to $f(x, y)$ belonging to $T P_{2}$, or $X$ and $Y$ are LRD.

The Morgenstern family belongs to $T P_{2}$ if $0 \leq \lambda \leq 1$. That is the family is either Positive Quadrant Dependent (PQD) or Negative Quadrant Dependent (NQD) [11].

From Equation (2.7),

$$
\frac{\partial^{2} \log f_{X, Y}(x, y)}{\partial x \partial y}=\frac{\left(\lambda_{3}-\lambda_{1} \lambda_{2}\right) f_{X}(x) f_{Y}(y)}{\left[1-\lambda_{1} F_{X}(x)-\lambda_{2} F_{Y}(y)+\lambda_{3} F_{X}(x) F_{Y}(y)\right]^{2}}
$$

showing that $f_{X, Y}(x, y)$ is $T P_{2}\left(R R_{2}\right)$ if $\lambda_{3} \geq(\leq) \lambda_{1} \lambda_{2}$. So Cambanis family belongs to $T P_{2}$ if $\lambda_{3} \geq \lambda_{1} \lambda_{2}$. And thus for concomitants of order statistics from the Morgenstern family,

$$
\lambda_{3}-\lambda_{1} \lambda_{2}=\lambda^{2}\left(\frac{4 r(n-s+1)}{(n+1)^{2}(n+2)}\right) \geq 0 .
$$

Hence $F_{[r, s: n]}\left(y_{1}, y_{2}\right)$ belongs to $T P_{2}$ or $Y_{[r: n]}$ and $Y_{[s: n]}$ are LRD for $-1 \leq \lambda \leq 1 ; 1 \leq r<s \leq n$.

It is directly established that $T P_{2}$ implies other dependency concepts such as stochastic increase (SI), right corner set increasing (RCSI), right (left) tail increasing (decreasing), association, PQD, weakly positive quadrant dependence and positive correlation (see [13,24]). Thus concomitants of order statistics from Morgenstern family belongs to $T P_{2} \Rightarrow P Q D$ and all other positive dependence concepts for $-1 \leq \lambda \leq 1$; $1 \leq r<s \leq n$.

There are a variety of ways to measure dependence. The most widely known scale-invariant measures of association are Kendall's tau and Spearman's rho. The dependence measurement for the Cambanis family is computed by Kendall's tau and is calculated by using Equations (2.4) and (2.7), as

$$
\begin{aligned}
\tau & =4 \int_{-\infty}^{\infty} \int_{-\infty}^{\infty} F_{X, Y}(x, y) f_{X, Y}(x, y) d x d y-1 \\
& =\frac{2}{9}\left(\lambda_{3}-\lambda_{1} \lambda_{2}\right)
\end{aligned}
$$

By substituting $\lambda_{1}, \lambda_{2}$ and $\lambda_{3}$ in Equation (2.9), the Kendall's tau for the $r^{\text {th }}$ and $s^{\text {th }}$ concomitants from the Morgenstern family is

$$
\tau=\frac{8}{9} \lambda^{2} \frac{r(n-s+1)}{(n+1)^{2}(n+2)} \geq 0 ; 1 \leq r<s \leq n .
$$

Using Equations (2.4-2.6), the Spearman's rho for the Cambanis family is calculated as

$$
\begin{aligned}
\rho & =12 \int_{-\infty}^{\infty} \int_{-\infty}^{\infty}\left[F_{X, Y}(x, y)-H_{X}(x) G_{Y}(y)\right] d H_{X}(x) d G_{Y}(y) \\
& =\frac{\left(\lambda_{3}-\lambda_{1} \lambda_{2}\right)}{3}
\end{aligned}
$$

By substituting $\lambda_{1}, \lambda_{2}$ and $\lambda_{3}$ in Equation (2.10), the Spearman's rho for the $r^{\text {th }}$ and $s^{\text {th }}$ concomitants from the Morgenstern family is

$$
\rho=\frac{4}{3} \lambda^{2} \frac{r(n-s+1)}{(n+1)^{2}(n+2)} \geq 0 ; 1 \leq r<s \leq n .
$$




\section{DISTRIBUTION THEORY OF LIFETIMES OF TWO COMPONENT SYSTEM USING CONCOMITANTS OF ORDER STATISTICS}

Let $F_{Y_{[r: n]}}$ and $F_{Y_{[s: n]}}$ denote the distribution function of the component lifetimes of the concomitants of order statistics $X_{r: n}$ and $X_{s: n}$ respectively. Let $T_{1}=\min \left(Y_{[r: n]}, Y_{[s: n]}\right)$, and $T_{2}=\max \left(Y_{[r: n]}, Y_{[s: n]}\right)$ denote the lifetimes of the series and parallel systems of two components, respectively. Let $F_{(i)}(t), R_{(i)}(t)$ and $f_{(i)}(t)$ denote the distribution function, survival function and density functions of $T_{i}, i=1,2$. The mean times to failure of $T_{1}$ and $T_{2}$ are denoted by $\mu_{(1)}$ and $\mu_{(2)}$, respectively.

From [11],

$$
\begin{aligned}
R_{(1)}(t) & =\bar{F}(t, t) \\
& =\operatorname{Pr}\left[Y_{[r: n]}>t, Y_{[s: n]}>t\right] \\
& =1-F_{Y_{[r, n]}}(t)-F_{Y_{[s, n]}}(t)+F_{[r, s: n]}(t, t) .
\end{aligned}
$$

Using the Equations (2.1), (2.3) and (3.1), we find

$$
R_{(1)}(t)=1-2 F(t)+[F(t)]^{2}-2 \delta_{\lambda} F(t)[1-F(t)]^{2}+\delta_{\lambda^{2}}[F(t)]^{2}[1-F(t)]^{2},
$$

where $\delta_{\lambda}=\lambda\left\{\frac{n-r-s+1}{n+1}\right\}$.

The corresponding density function can be obtained from Equation (3.2) as,

$$
\begin{aligned}
f_{(1)}(t)= & \frac{-\partial}{\partial t}\left(R_{(1)}(t)\right) \\
= & 2 f(t)-2 F(t) f(t)+2 \delta_{\lambda} f(t)\left\{1-4 F(t)+3[F(t)]^{2}\right\} \\
& -2 \delta_{\lambda^{2}} F(t) f(t)\left\{1-3 F(t)+2[F(t)]^{2}\right\} .
\end{aligned}
$$

Using the formula for the density of order statistics in (3.3), we find

$$
\begin{aligned}
f_{(1)}(t)= & 2 f(t)-f_{2: 2}(t)+2 \delta_{\lambda}\left[f(t)-2 f_{2: 2}(t)+f_{3: 3}(t)\right] \\
& -\delta_{\lambda^{2}}\left[f_{2: 2}(t)-2 f_{3: 3}(t)+f_{4: 4}(t)\right],
\end{aligned}
$$

where $f_{r: n}(t)$ is the density of $Y_{r: n}$.

From [11],

$$
f_{(1)}(t)+f_{(2)}(t)=f_{Y_{[r: n]}}(t)+f_{Y_{[s: n]}}(t) \text {. }
$$

Using Equations (2.2), (3.3) and (3.5), we get

$$
f_{(2)}(t)=2 F(t) f(t)+2 \delta_{\lambda} F(t) f(t)[2-3 F(t)]+2 \delta_{\lambda^{2}} F(t) f(t)\left[1-3 F(t)+2[F(t)]^{2}\right] .
$$

Using the formula for the density of order statistics in (3.6), we find

$$
f_{(2)}(t)=f_{2: 2}(t)+2 \delta_{\lambda}\left[f_{2: 2}(t)-f_{3: 3}(t)\right]+\delta_{\lambda^{2}}\left[f_{2: 2}(t)-2 f_{3: 3}(t)+f_{4: 4}(t)\right] .
$$

Moments of $T_{1}$ and $T_{2}$

From (3.4), the $k^{\text {th }}$ moment of $T_{1}$ can be derived as

$$
\begin{aligned}
\mu_{(1)}^{(k)}(t) & =\int t^{k} f_{(1)}(t) d t \\
& =2 \mu^{(k)}-\mu_{2: 2}^{(k)}+2 \delta_{\lambda}\left[\mu^{(k)}-2 \mu_{2: 2}^{(k)}+\mu_{3: 3}^{(k)}\right]-\delta_{\lambda^{2}}\left[\mu_{2: 2}^{(k)}-2 \mu_{3: 3}^{(k)}+\mu_{4: 4}^{(k)}\right] .
\end{aligned}
$$


The $k^{\text {th }}$ moment of $T_{2}$ follows from (3.7) as,

$$
\begin{aligned}
\mu_{(2)}^{(k)}(t) & =\int t^{k} f_{(2)}(t) d t \\
& =\mu_{2: 2}^{(k)}+2 \delta_{\lambda}\left[\mu_{2: 2}^{(k)}-\mu_{3: 3}^{(k)}\right]+\delta_{\lambda^{2}}\left[\mu_{2: 2}^{(k)}-2 \mu_{3: 3}^{(k)}+\mu_{4: 4}^{(k)}\right],
\end{aligned}
$$

where $\mu^{(k)}=E\left[Y^{k}\right]$ and $\mu_{r: n}^{(k)}=E\left[Y_{r: n}^{k}\right]$.

\section{APPLICATION IN RELIABILITY}

Gupta and Kundu [25] introduced the exponentiated exponential (EE) distribution as a generalization of the standard exponential distribution with corresponding cdf and pdf are respectively,

$$
F_{X}(x)=\left(1-e^{-\theta x}\right)^{\alpha} ; x>0, \theta>0, \alpha>0,
$$

and

$$
f_{X}(x)=\alpha \theta e^{-\theta x}\left(1-e^{-\theta x}\right)^{\alpha-1} ; x>0, \theta>0, \alpha>0
$$

We denote the EE distribution with parameters $\theta$ and $\alpha$ as $E E(\theta, \alpha)$. Gupta and Kundu [25] showed that the mean, variance and moment generating function of the random variable $X$ with $E E(\theta, \alpha)$ can be given as

$$
\begin{aligned}
E(X) & =\frac{1}{\theta}[\psi(\alpha+1)-\psi(1)], \\
\operatorname{Var}(X) & =\frac{1}{\theta^{2}}\left[\psi^{\prime}(1)-\psi^{\prime}(\alpha+1)\right],
\end{aligned}
$$

and

$$
M_{X}(t)=\alpha \operatorname{Beta}(\alpha, 1-t / \theta)
$$

where $\psi($.$) is the digamma function, \psi^{\prime}($.$) is its derivative, and \operatorname{Beta}(a, b)=\frac{\Gamma(a) \Gamma(b)}{\Gamma(a+b)}$.

Let $Y$ be the life time of a very expensive component of a two component system and $X$ be an inexpensive variable (directly measurable or observable) which is correlated with $Y$. Suppose $(X, Y)$ follows MTBEED. Then from (1.1), the cdf of $(X, Y)$ is,

$$
\begin{array}{r}
F_{X, Y}(x, y)=\left(1-e^{-\theta_{1} x}\right)^{\alpha_{1}}\left(1-e^{-\theta_{2} y}\right)^{\alpha_{2}}\left\{1+\lambda\left[1-\left(1-e^{-\theta_{1} x}\right)^{\alpha_{1}}\right]\left[1-\left(1-e^{-\theta_{2} y}\right)^{\alpha_{2}}\right]\right\}, \\
x, y>0, \theta_{1}, \theta_{2}, \alpha_{1}, \alpha_{2}>0,-1 \leq \lambda \leq 1,
\end{array}
$$

Let $\left(X_{r: n}, X_{s: n}\right),(r<s)$ be the pair of $r^{\text {th }}$ and $s^{\text {th }}$ order statistics of the inexpensive variable $X$ and $\left(Y_{[r: n]}, Y_{[s: n]}\right)$ be the associated $Y$ measurements or concomitants. Then the $\operatorname{cdf}$ of $\left(Y_{[r: n]}, Y_{[s: n]}\right)$ follows from Equation (2.3) as,

$$
\begin{aligned}
F_{[r, s: n]}\left(y_{1}, y_{2}\right)= & \left(1-e^{-\theta_{2} y_{1}}\right)^{\alpha_{2}}\left(1-e^{-\theta_{2} y_{2}}\right)^{\alpha_{2}}\left\{1+\delta_{\lambda_{r}}\left[1-\left(1-e^{-\theta_{2} y_{1}}\right)^{\alpha_{2}}\right]\right. \\
& \left.+\delta_{\lambda_{s}}\left[1-\left(1-e^{-\theta_{2} y_{2}}\right)^{\alpha_{2}}\right]+\delta_{\lambda^{2}}\left[1-\left(1-e^{-\theta_{2} y_{1}}\right)^{\alpha_{2}}\right]\left[1-\left(1-e^{-\theta_{2} y_{2}}\right)^{\alpha_{2}}\right]\right\} .
\end{aligned}
$$

It follows from (3.3) and (3.6) that the density function of the lifetime of a series, and parallel systems using the expensive components $Y_{[r: n]}$ and $Y_{[s: n]}$ are, respectively

$$
\begin{aligned}
f_{(1)}(t)= & 2\left(1+\delta_{\lambda}\right) \alpha_{2} \theta_{2} e^{-\theta_{2} t}\left(1-e^{-\theta_{2} t}\right)^{\alpha_{2}-1}-2 \alpha_{2} \theta_{2} e^{-\theta_{2} t}\left(1-e^{-\theta_{2} t}\right)^{2 \alpha_{2}-1}\left(1+4 \delta_{\lambda}+\delta_{\lambda^{2}}\right) \\
& +6 \alpha_{2} \theta_{2} e^{-\theta_{2} t}\left(1-e^{-\theta_{2} t}\right)^{3 \alpha_{2}-1}\left(\delta_{\lambda}+\delta_{\lambda^{2}}\right)-4 \delta_{\lambda^{2}} \alpha_{2} \theta_{2} e^{-\theta_{2} t}\left(1-e^{-\theta_{2} t}\right)^{4 \alpha_{2}-1}
\end{aligned}
$$

and

$$
\begin{aligned}
f_{(2)}(t)= & 2\left(1+2 \delta_{\lambda}+\delta_{\lambda^{2}}\right) \alpha_{2} \theta_{2} e^{-\theta_{2} t}\left(1-e^{-\theta_{2} t}\right)^{2 \alpha_{2}-1} \\
& -6\left(\delta_{\lambda}+\delta_{\lambda^{2}}\right) \alpha_{2} \theta_{2} e^{-\theta_{2} t}\left(1-e^{-\theta_{2} t}\right)^{3 \alpha_{2}-1}+4 \delta_{\lambda^{2}} \alpha_{2} \theta_{2} e^{-\theta_{2} t}\left(1-e^{-\theta_{2} t}\right)^{4 \alpha_{2}-1} .
\end{aligned}
$$


It follows from (4.6) and (4.7) that

$$
f_{(1)}(t)=2\left(1+\delta_{\lambda}\right) f_{U}(t)-f_{V}(t)\left(1+4 \delta_{\lambda}+\delta_{\lambda^{2}}\right)+2\left(\delta_{\lambda}+\delta_{\lambda^{2}}\right) f_{W}(t)-\delta_{\lambda^{2}} f_{Z}(t),
$$

and

$$
f_{(2)}(t)=\left(1+2 \delta_{\lambda}+\delta_{\lambda^{2}}\right) f_{V}(t)-2\left(\delta_{\lambda}+\delta_{\lambda^{2}}\right) f_{W}(t)+\delta_{\lambda^{2}} f_{Z}(t),
$$

where $f_{U}(t), f_{V}(t), f_{W}(t)$ and $f_{Z}(t)$ are pdf's of random variables $U, V, W$ and $Z$ with $E E\left(\theta_{2}, \alpha_{2}\right), E E\left(\theta_{2}, 2 \alpha_{2}\right), E E\left(\theta_{2}, 3 \alpha_{2}\right)$ and $E E\left(\theta_{2}, 4 \alpha_{2}\right)$ respectively.

The $k^{\text {th }}$ moment of $T_{1}$ and $T_{2}$ follows from (4.8) and (4.9), respectively as

$$
\begin{aligned}
\mu_{(1)}^{(k)}(t)= & 2\left(1+\delta_{\lambda}\right) E\left[U^{k}\right]-\left(1+4 \delta_{\lambda}+\delta_{\lambda^{2}}\right) E\left[V^{k}\right] \\
& +2\left(\delta_{\lambda}+\delta_{\lambda^{2}}\right) E\left[W^{k}\right]-\delta_{\lambda^{2}} E\left[Z^{k}\right],
\end{aligned}
$$

and

$$
\mu_{(2)}^{(k)}(t)=\left(1+2 \delta_{\lambda}+\delta_{\lambda^{2}}\right) E\left[V^{k}\right]-2\left(\delta_{\lambda}+\delta_{\lambda^{2}}\right) E\left[W^{k}\right]+\delta_{\lambda^{2}} E\left[Z^{k}\right]
$$

The mean times to failure (MTTF) of the corresponding systems are obtained by setting $k=1$ in (4.10) and (4.11), and using Equation (4.3). They are, respectively

$$
\begin{aligned}
\mu_{(1)}(t)= & \frac{1}{\theta_{2}}\left\{2 \psi\left(\alpha_{2}+1\right)-\psi\left(2 \alpha_{2}+1\right)-\psi(1)+2 \delta_{\lambda}\left[\psi\left(\alpha_{2}+1\right)-2 \psi\left(2 \alpha_{2}+1\right)+\psi\left(3 \alpha_{2}+1\right)\right]\right. \\
& \left.-\delta_{\lambda^{2}}\left[\psi\left(2 \alpha_{2}+1\right)-2 \psi\left(3 \alpha_{2}+1\right)+\psi\left(4 \alpha_{2}+1\right)\right]\right\},
\end{aligned}
$$

and

$$
\begin{aligned}
\mu_{(2)}(t)= & \frac{1}{\theta_{2}}\left\{\psi\left(2 \alpha_{2}+1\right)-\psi(1)+2 \delta_{\lambda}\left[\psi\left(2 \alpha_{2}+1\right)-\psi\left(3 \alpha_{2}+1\right)\right]\right. \\
& \left.+\delta_{\lambda^{2}}\left[\psi\left(2 \alpha_{2}+1\right)-2 \psi\left(3 \alpha_{2}+1\right)+\psi\left(4 \alpha_{2}+1\right)\right]\right\} .
\end{aligned}
$$

\begin{tabular}{|c|c|c|c|c|c|c|c|c|}
\hline \multirow[t]{2}{*}{$\mathrm{n}$} & \multirow[t]{2}{*}{$\alpha_{2}$} & \multirow[t]{2}{*}{$\lambda$} & \multicolumn{6}{|c|}{$\theta_{2} \mu_{(1)}(t)$} \\
\hline & & & $(r, s)=(1,2)$ & $(r, s)=(1,3)$ & $(r, s)=(2,3)$ & $(r, s)=(n-2, n-1)$ & $(r, s)=(n-2, n)$ & $(\mathbf{r}, \mathbf{s})=(\mathbf{n}-1, \mathbf{n})$ \\
\hline \multirow[t]{14}{*}{10} & 1 & -1 & 0.7879 & 0.7449 & 0.7096 & 0.3460 & 0.3207 & 0.3031 \\
\hline & & -.6 & 0.6618 & 0.6391 & 0.6191 & 0.4009 & 0.3846 & 0.3709 \\
\hline & & -.2 & 0.5503 & 0.5437 & 0.5375 & 0.4647 & 0.4589 & 0.4533 \\
\hline & & .2 & 0.4533 & 0.4589 & 0.4647 & 0.5375 & 0.5437 & 0.5503 \\
\hline & & .6 & 0.3709 & 0.3846 & 0.4009 & 0.6191 & 0.6391 & 0.6618 \\
\hline & & 1 & 0.3031 & 0.3207 & 0.3460 & 0.7096 & 0.7449 & 0.7879 \\
\hline & 2 & -1 & 1.2857 & 1.2314 & 1.1860 & 0.7132 & 0.6798 & 0.6554 \\
\hline & & -.6 & 1.1252 & 1.0961 & 1.0703 & 0.7867 & 0.7652 & 0.7470 \\
\hline & & -.2 & 0.9819 & 0.9734 & 0.9653 & 0.8707 & 0.8631 & 0.8558 \\
\hline & & .2 & 0.8558 & 0.8631 & 0.8707 & 0.9653 & 0.9734 & 0.9819 \\
\hline & & .6 & 0.7470 & 0.7652 & 0.7867 & 1.0703 & 1.0961 & 1.1252 \\
\hline & & 1 & 0.6554 & 0.6798 & 0.7132 & 1.1860 & 1.2314 & 1.2857 \\
\hline & 3 & -1 & 1.6195 & 1.5605 & 1.5109 & 0.9923 & 0.9554 & 0.9281 \\
\hline & & -.6 & 1.4447 & 1.4130 & 1.3848 & 1.0737 & 1.0500 & 1.0298 \\
\hline
\end{tabular}

The following relations are directly from (4.12) and (4.13).

Relation $4.1 \mu_{(1)[r, s: n]}(-\lambda)=\mu_{(1)[n-s+1, n-r+1: n]}(\lambda)$.

Relation $4.2 \mu_{(2)[r, s: n]}(-\lambda)=\mu_{(2)[n-s+1, n-r+1: n]}(\lambda)$.

The $\theta_{2}$ times MTTF of series and parallel systems of a two component systems using concomitants are tabulated in Tables 1 and 2 for specific values of $n, r, s, \alpha_{2}$ and $\lambda$.

Table $1 \mid \theta_{2}$ times MTTF of two component series system using concomitants. 
Table 1 的 times MTTF of two component series system using concomitants. (Continued)

\begin{tabular}{|c|c|c|c|c|c|c|c|c|}
\hline \multirow[t]{2}{*}{$\mathbf{n}$} & \multirow[t]{2}{*}{$\alpha_{2}$} & \multirow[t]{2}{*}{$\lambda$} & \multicolumn{5}{|c|}{$\theta_{2} \mu_{(1)}(t)$} & \multirow[b]{2}{*}{$(\mathbf{r}, \mathbf{s})=(\mathbf{n}-1, \mathbf{n})$} \\
\hline & & & $(r, s)=(1,2)$ & $(r, s)=(1,3)$ & $(r, s)=(2,3)$ & $(\mathrm{r}, \mathrm{s})=(\mathrm{n}-2, \mathrm{n}-1)$ & $(r, s)=(n-2, n)$ & \\
\hline & & -.2 & 1.2881 & 1.2788 & 1.2699 & 1.1662 & 1.1578 & 1.1498 \\
\hline & & .2 & 1.1498 & 1.1578 & 1.1662 & 1.2699 & 1.2788 & 1.2881 \\
\hline & & .6 & 1.0298 & 1.0500 & 1.0737 & 1.3848 & 1.4130 & 1.4447 \\
\hline & & 1 & 0.9281 & 0.9554 & 0.9923 & 1.5109 & 1.5605 & 1.6195 \\
\hline & 4 & -1 & 1.8701 & 1.8084 & 1.7566 & 1.2129 & 1.1742 & 1.1452 \\
\hline & & -.6 & 1.6874 & 1.6544 & 1.6248 & 1.2987 & 1.2738 & 1.2525 \\
\hline & & -.2 & 1.5237 & 1.5139 & 1.5046 & 1.3959 & 1.3871 & 1.3787 \\
\hline & & .2 & 1.3787 & 1.3871 & 1.3959 & 1.5046 & 1.5139 & 1.5237 \\
\hline & & .6 & 1.2525 & 1.2738 & 1.2987 & 1.6248 & 1.6544 & 1.6874 \\
\hline & & 1 & 1.1452 & 1.1742 & 1.2129 & 1.7566 & 1.8084 & 1.8701 \\
\hline & 5 & -1 & 2.0705 & 2.0072 & 1.9540 & 1.3946 & 1.3546 & 1.3247 \\
\hline & & -.6 & 1.8830 & 1.8490 & 1.8187 & 1.4831 & 1.4575 & 1.4355 \\
\hline & & -.2 & 1.7147 & 1.7047 & 1.6951 & 1.5832 & 1.5742 & 1.5655 \\
\hline & & .2 & 1.5655 & 1.5742 & 1.5832 & 1.6951 & 1.7047 & 1.7147 \\
\hline & & .6 & 1.4355 & 1.4575 & 1.4831 & 1.8187 & 1.8490 & 1.8830 \\
\hline & & 1 & 1.3247 & 1.3546 & 1.3946 & 1.9540 & 2.0072 & 2.0705 \\
\hline \multirow[t]{30}{*}{20} & 1 & -1 & 0.8474 & 0.8243 & 0.8034 & 0.2955 & 0.2847 & 0.2760 \\
\hline & & -.6 & 0.6936 & 0.6815 & 0.6702 & 0.3654 & 0.3577 & 0.3508 \\
\hline & & -.2 & 0.5596 & 0.5562 & 0.5528 & 0.4512 & 0.4482 & 0.4453 \\
\hline & & .2 & 0.4453 & 0.4482 & 0.4512 & 0.5528 & 0.5562 & 0.5596 \\
\hline & & .6 & 0.3508 & 0.3577 & 0.3654 & 0.6702 & 0.6815 & 0.6936 \\
\hline & & 1 & 0.2760 & 0.2847 & 0.2955 & 0.8034 & 0.8243 & 0.8474 \\
\hline & 2 & -1 & 1.3612 & 1.3321 & 1.3054 & 0.6451 & 0.6305 & 0.6184 \\
\hline & & -.6 & 1.1659 & 1.1504 & 1.1359 & 0.7397 & 0.7294 & 0.7201 \\
\hline & & -.2 & 0.9939 & 0.9894 & 0.9850 & 0.8530 & 0.8491 & 0.8453 \\
\hline & & .2 & 0.8453 & 0.8491 & 0.8530 & 0.9850 & 0.9894 & 0.9939 \\
\hline & & .6 & 0.7201 & 0.7294 & 0.7397 & 1.1359 & 1.1504 & 1.1659 \\
\hline & & 1 & 0.6184 & 0.6305 & 0.6451 & 1.3054 & 1.3321 & 1.3612 \\
\hline & 3 & -1 & 1.7017 & 1.6699 & 1.6410 & 0.9166 & 0.9003 & 0.8867 \\
\hline & & -.6 & 1.4891 & 1.4722 & 1.4564 & 1.0217 & 1.0104 & 1.0000 \\
\hline & & -.2 & 1.3013 & 1.2964 & 1.2916 & 1.1467 & 1.1424 & 1.1383 \\
\hline & & .2 & 1.1383 & 1.1424 & 1.1467 & 1.2916 & 1.2964 & 1.3013 \\
\hline & & .6 & 1.0000 & 1.0104 & 1.0217 & 1.4564 & 1.4722 & 1.4891 \\
\hline & & 1 & 0.8867 & 0.9003 & 0.9166 & 1.6410 & 1.6699 & 1.7017 \\
\hline & 4 & -1 & 1.9558 & 1.9227 & 1.8925 & 1.1331 & 1.1159 & 1.1015 \\
\hline & & -.6 & 1.7338 & 1.7162 & 1.6997 & 1.2440 & 1.2321 & 1.2213 \\
\hline & & -.2 & 1.5374 & 1.5323 & 1.5273 & 1.3754 & 1.3709 & 1.3666 \\
\hline & & .2 & 1.3666 & 1.3709 & 1.3754 & 1.5273 & 1.5323 & 1.5374 \\
\hline & & .6 & 1.2213 & 1.2321 & 1.2440 & 1.6997 & 1.7162 & 1.7338 \\
\hline & & 1 & 1.1015 & 1.1159 & 1.1331 & 1.8925 & 1.9227 & 1.9558 \\
\hline & 5 & -1 & 2.1584 & 2.1245 & 2.0935 & 1.3121 & 1.2944 & 1.2794 \\
\hline & & -.6 & 1.9307 & 1.9126 & 1.8955 & 1.4267 & 1.4145 & 1.4033 \\
\hline & & -.2 & 1.7289 & 1.7236 & 1.7184 & 1.5622 & 1.5576 & 1.5531 \\
\hline & & .2 & 1.5531 & 1.5576 & 1.5622 & 1.7184 & 1.7236 & 1.7289 \\
\hline & & .6 & 1.4033 & 1.4145 & 1.4267 & 1.8955 & 1.9126 & 1.9307 \\
\hline & & 1 & 1.2794 & 1.2944 & 1.3121 & 2.0935 & 2.1245 & 2.1584 \\
\hline \multirow[t]{9}{*}{30} & 1 & -1 & 0.8693 & 0.8535 & 0.8387 & 0.2796 & 0.2729 & 0.2672 \\
\hline & & -.6 & 0.7052 & 0.6969 & 0.6890 & 0.3536 & 0.3486 & 0.3439 \\
\hline & & -.2 & 0.5629 & 0.5606 & 0.5583 & 0.4465 & 0.4445 & 0.4425 \\
\hline & & .2 & 0.4425 & 0.4445 & 0.4465 & 0.5583 & 0.5606 & 0.5629 \\
\hline & & .6 & 0.3439 & 0.3486 & 0.3536 & 0.6890 & 0.6969 & 0.7052 \\
\hline & & 1 & 0.2672 & 0.2729 & 0.2796 & 0.8387 & 0.8535 & 0.8693 \\
\hline & 2 & -1 & 1.3889 & 1.3690 & 1.3502 & 0.6234 & 0.6142 & 0.6062 \\
\hline & & -.6 & 1.1806 & 1.1701 & 1.1599 & 0.7238 & 0.7172 & 0.7109 \\
\hline & & -.2 & 0.9982 & 0.9951 & 0.9922 & 0.8468 & 0.8442 & 0.8416 \\
\hline
\end{tabular}


Table 1 期 times MTTF of two component series system using concomitants. (Continued)

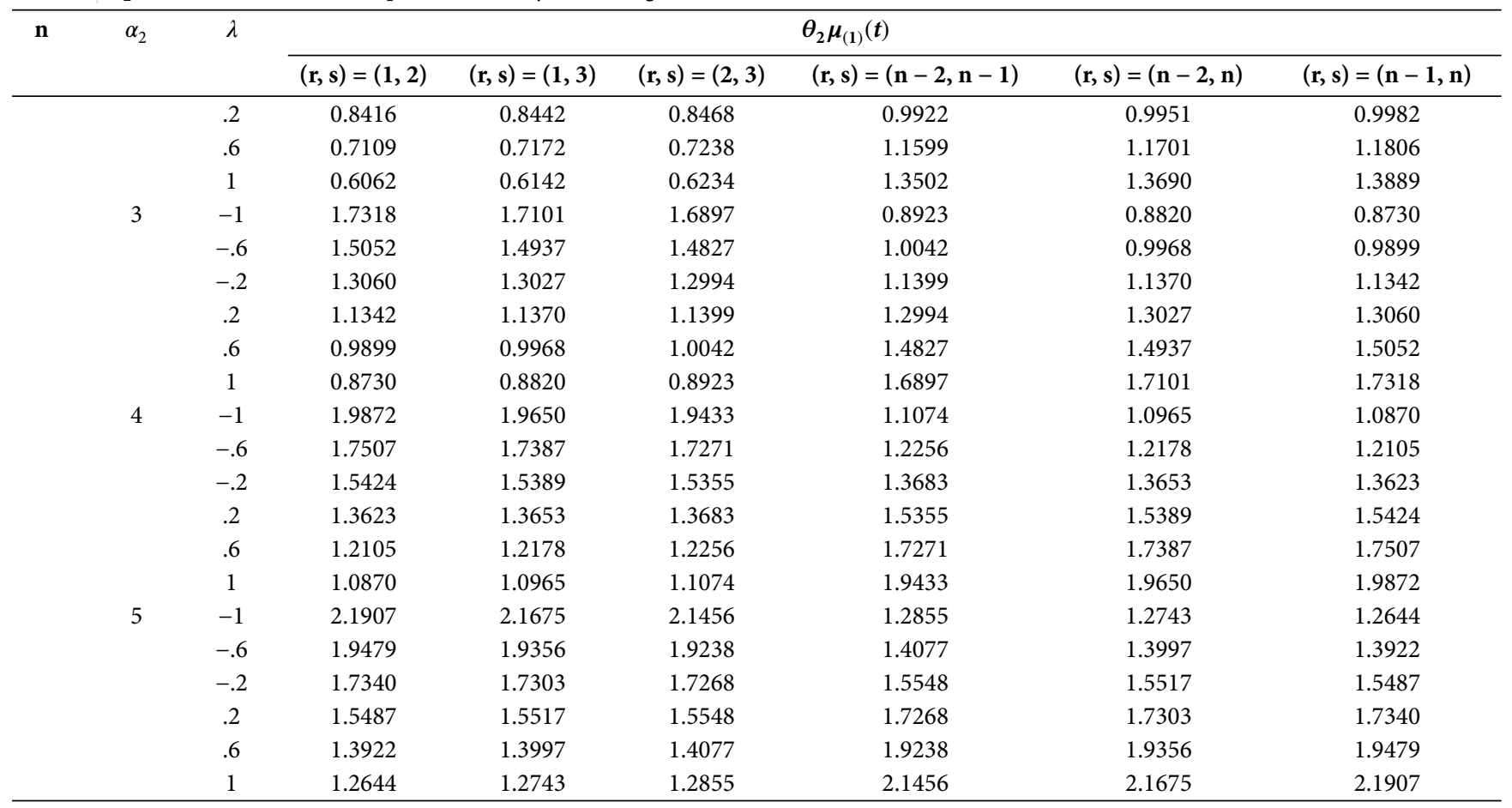

MTTF, mean times to failure.

Table $2 \theta_{2}$ times MTTF of two component parallel system using concomitants.

\begin{tabular}{|c|c|c|c|c|c|c|c|c|}
\hline \multirow{3}{*}{$\mathbf{n}$} & \multirow{3}{*}{$\alpha_{2}$} & \multicolumn{7}{|c|}{$\theta_{2} \mu_{(2)}(t)$} \\
\hline & & \multirow[t]{2}{*}{$\lambda$} & \multicolumn{5}{|c|}{$(r, s)$} & \multirow[b]{2}{*}{$(n-1, n)$} \\
\hline & & & $(1,2)$ & $(1,3)$ & $(2,3)$ & $(n-2, n-1)$ & $(n-2, n)$ & \\
\hline \multirow[t]{26}{*}{10} & 1 & -1 & 1.9394 & 1.8914 & 1.8359 & 1.1086 & 1.0429 & 0.9697 \\
\hline & & -.6 & 1.7746 & 1.7427 & 1.7082 & 1.2718 & 1.2336 & 1.1927 \\
\hline & & -.2 & 1.5952 & 1.5835 & 1.5716 & 1.4262 & 1.4138 & 1.4012 \\
\hline & & .2 & 1.4012 & 1.4138 & 1.4262 & 1.5716 & 1.5835 & 1.5952 \\
\hline & & 6 & 1.1927 & 1.2336 & 1.2718 & 1.7082 & 1.7427 & 1.7746 \\
\hline & & 1 & 0.9697 & 1.0429 & 1.1086 & 1.8359 & 1.8914 & 1.9394 \\
\hline & 2 & -1 & 2.5628 & 2.5111 & 2.4504 & 1.6504 & 1.5777 & 1.4961 \\
\hline & & -.6 & 2.3840 & 2.3493 & 2.3115 & 1.8315 & 1.7893 & 1.7439 \\
\hline & & -.2 & 2.1878 & 2.1751 & 2.1620 & 2.0020 & 1.9884 & 1.9745 \\
\hline & & .2 & 1.9745 & 1.9884 & 2.0020 & 2.1620 & 2.1751 & 2.1878 \\
\hline & & 6 & 1.7439 & 1.7893 & 1.8315 & 2.3115 & 2.3493 & 2.3840 \\
\hline & & 1 & 1.4961 & 1.5777 & 1.6504 & 2.4504 & 2.5111 & 2.5628 \\
\hline & 3 & -1 & 2.9441 & 2.8911 & 2.8285 & 2.0017 & 1.9264 & 1.8417 \\
\hline & & -.6 & 2.7602 & 2.7245 & 2.6855 & 2.1894 & 2.1458 & 2.0987 \\
\hline & & -.2 & 2.5580 & 2.5448 & 2.5313 & 2.3659 & 2.3519 & 2.3375 \\
\hline & & .2 & 2.3375 & 2.3519 & 2.3659 & 2.5313 & 2.5448 & 2.5580 \\
\hline & & .6 & 2.0987 & 2.1458 & 2.1894 & 2.6855 & 2.7245 & 2.7602 \\
\hline & & 1 & 1.8417 & 1.9264 & 2.0017 & 2.8285 & 2.8911 & 2.9441 \\
\hline & 4 & -1 & 3.2196 & 3.1658 & 3.1023 & 2.2615 & 2.1849 & 2.0985 \\
\hline & & -.6 & 3.0330 & 2.9968 & 2.9572 & 2.4527 & 2.4083 & 2.3604 \\
\hline & & -.2 & 2.8276 & 2.8143 & 2.8005 & 2.6323 & 2.6181 & 2.6034 \\
\hline & & .2 & 2.6034 & 2.6181 & 2.6323 & 2.8005 & 2.8143 & 2.8276 \\
\hline & & .6 & 2.3604 & 2.4083 & 2.4527 & 2.9572 & 2.9968 & 3.0330 \\
\hline & & 1 & 2.0985 & 2.1849 & 2.2615 & 3.1023 & 3.1658 & 3.2196 \\
\hline & 5 & -1 & 3.4353 & 3.3812 & 3.3170 & 2.4677 & 2.3903 & 2.3029 \\
\hline & & -.6 & 3.2471 & 3.2107 & 3.1706 & 2.6610 & 2.6162 & 2.5677 \\
\hline
\end{tabular}


Table $2 \theta_{2}$ times MTTF of two component parallel system using concomitants. (Continued)

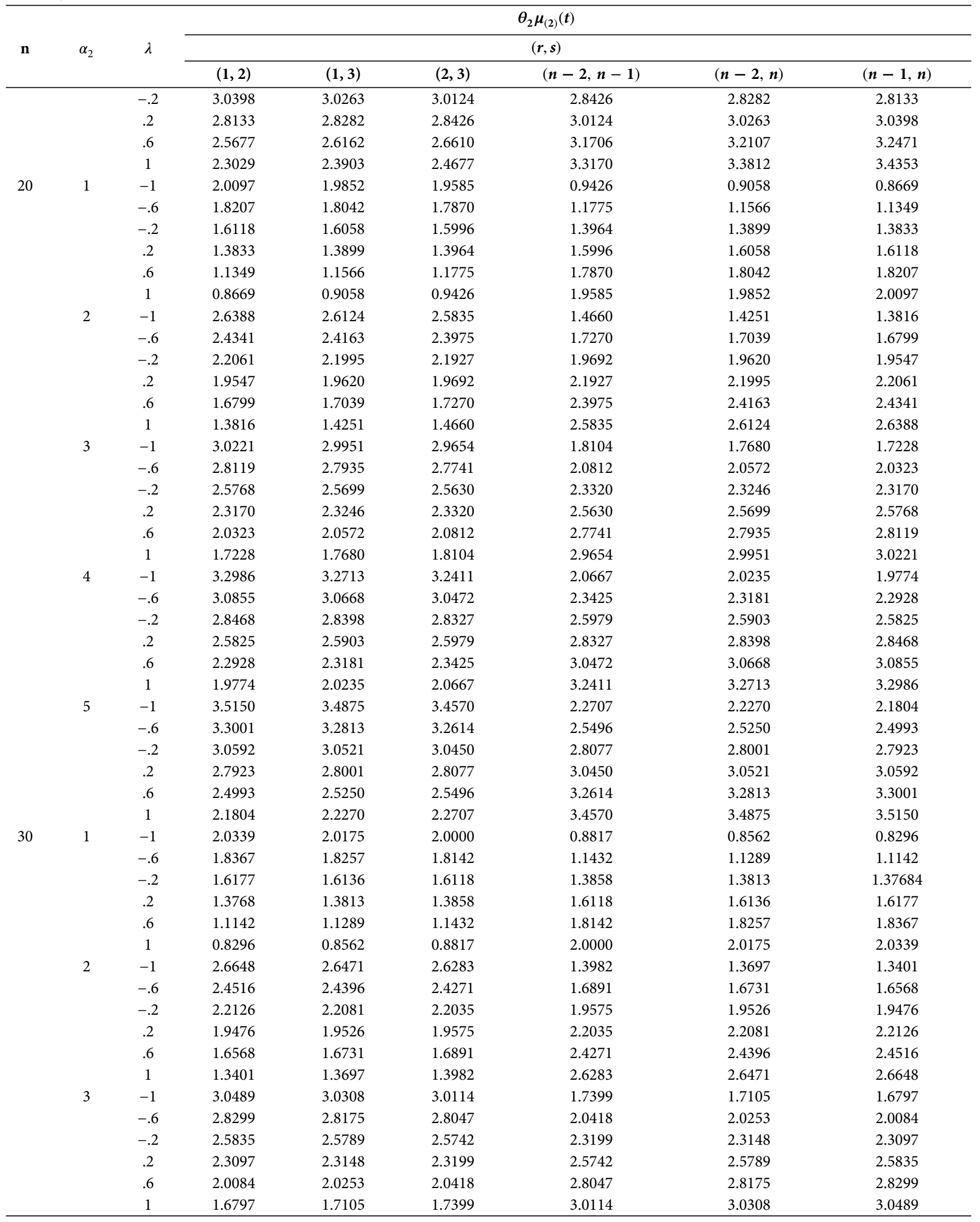


Table $2 \theta_{2}$ times MTTF of two component parallel system using concomitants. (Continued)

\begin{tabular}{|c|c|c|c|c|c|c|c|c|}
\hline \multirow{3}{*}{$\mathbf{n}$} & \multirow{3}{*}{$\alpha_{2}$} & \multicolumn{7}{|c|}{$\overline{\theta_{2} \mu_{(2)}(t)}$} \\
\hline & & \multirow[t]{2}{*}{$\lambda$} & \multicolumn{6}{|c|}{$(\boldsymbol{r}, \boldsymbol{s})$} \\
\hline & & & $(1,2)$ & $(1,3)$ & $(2,3)$ & $(n-2, n-1)$ & $(n-2, n)$ & $(n-1, n)$ \\
\hline & \multirow[t]{6}{*}{4} & -1 & 3.3257 & 3.3074 & 3.2877 & 1.9949 & 1.9649 & 1.9335 \\
\hline & & -.6 & 3.1038 & 3.0912 & 3.0782 & 2.3025 & 2.2857 & 2.2684 \\
\hline & & -.2 & 2.8536 & 2.8488 & 2.8441 & 2.5855 & 2.5803 & 2.5751 \\
\hline & & .2 & 2.5751 & 2.5803 & 2.5855 & 2.8441 & 2.8488 & 2.8536 \\
\hline & & .6 & 2.2684 & 2.2857 & 2.3025 & 3.0782 & 3.0912 & 3.1038 \\
\hline & & 1 & 1.9335 & 1.9649 & 1.9949 & 3.2877 & 3.3074 & 3.3257 \\
\hline & \multirow[t]{6}{*}{5} & -1 & 3.5423 & 3.5238 & 3.5040 & 2.1981 & 2.1677 & 2.1360 \\
\hline & & -.6 & 3.3185 & 3.3059 & 3.2927 & 2.5092 & 2.4922 & 2.4747 \\
\hline & & -.2 & 3.0660 & 3.0613 & 3.0564 & 2.7953 & 2.7900 & 2.7847 \\
\hline & & .2 & 2.7847 & 2.7900 & 2.7953 & 3.0564 & 3.0613 & 3.0660 \\
\hline & & .6 & 2.4747 & 2.4922 & 2.5092 & 3.2927 & 3.3059 & 3.3185 \\
\hline & & 1 & 2.1360 & 2.1677 & 2.1981 & 3.5040 & 3.5238 & 3.5423 \\
\hline
\end{tabular}

MTTF, mean times to failure.

Table 3 Independent case.

\begin{tabular}{cccccc}
\hline \multicolumn{5}{c}{ Series System, $\theta_{2} \mu_{1}^{\prime}(t)$} \\
\hline$\alpha_{2}$ & 1 & 2 & 3 & 4 & 5 \\
& 0.5000 & 0.7500 & 0.9167 & 1.0417 & 1.1417 \\
\hline \multicolumn{5}{c}{ Parallel System, $\theta_{2} \mu_{2}^{\prime}(t)$} \\
\hline$\alpha_{2}$ & 1 & 2 & 3 & 4 & 5 \\
& 1.5000 & 2.2500 & 2.7500 & 3.1250 & 3.4250 \\
\hline
\end{tabular}

The MTTF of a series and parallel system based on independent components are respectively,

$$
\mu_{1}^{\prime}(t)=\frac{\mu_{x} \mu_{y}}{\mu_{x}+\mu_{y}},
$$

and

$$
\mu_{2}^{\prime}(t)=\mu_{x}+\mu_{y}-\frac{\mu_{x} \mu_{y}}{\mu_{x}+\mu_{y}},
$$

see [11]. The $\theta_{2}$ times MTTF of a series and parallel systems based on independent components for $\alpha_{2}=1,2,3,4$ and 5 are tabulated in Table 3.

The following conclusions are evident from the above Tables.

Comparing Tables 1 and 3, we can say that the selection of components in the series system based on concomitants will substantially increase the MTTF. It follows from Table 1 that if $\lambda<0$, the MTTF is optimal for the pair $r=1$, and $s=2$. On the otherhand, if $\lambda>0$, the MTTF is optimal for the pair $r=n-1$, and $s=n$. It is also observed that the MTTF of series system increases if $\lambda$, and $n$ increases numerically. The percentage relative gains in MTTF of a series system using the first and second concomitants over independent cases are tabulated in Table 4 for $\lambda=-0.2,-0.6$ and $-1 ; \alpha_{2}=1,2,3,4$ and 5; and $n=10,20$ and 30 .

Similarly, when comparing Tables 2 and 3, we can say that the selection of components in the parallel system based on concomitants will substantially increase the MTTF. It follows from Table 2 that if $\lambda=-1, \alpha_{2}=1,2,3,4$ and 5, the MTTF is optimal for the pair $r=1$, and $s$ $=2$. On the otherhand, if $\lambda=1, \alpha_{2}=1,2,3,4$ and 5, the MTTF is optimal for the pair $r=n-1$, and $s=n$. The percentage relative gains in MTTF of a parallel system using the first and second concomitants over independent cases are tabulated in Table 5 for $\lambda=-1 ; n=10,20$ and 30 and $\alpha_{2}=1,2,3,4$ and 5 .

Using relations (4.1) and (4.2), we deduce that the percentage relative gain in the MTTF of series and parallel systems using the $(n-1)^{\text {th }}$ and $n^{\text {th }}$ concomitants over independent case are the same as that of the above table by replacing $\lambda$ with $-\lambda$.

Thus we conclude that the design of two component series or parallel systems using concomitants substantially increases the MTTF of both the systems. Moreover, the selection of components based on concomitants is more effective in series systems than parallel systems. If $\lambda= \pm 1, \alpha_{2} \geq 5$ and $n \geq 30$, the percentage relative increase in the MTTF of a series system is greater than ninety one. Similarly, if $\lambda= \pm 1$, $\alpha_{2}=1$ and $n \geq 30$, the percentage relative increase in the MTTF of a parallel system is greater than thirty five. 
Table 4 The percentage relative gain in MTTF of a series system.

\begin{tabular}{cccccccc}
\hline & & \multicolumn{6}{c}{ Series System } \\
\cline { 4 - 7 } $\boldsymbol{n}$ & $\lambda$ & $\alpha_{2}$ & $\mathbf{1}$ & $\mathbf{2}$ & $\mathbf{3}$ & $\mathbf{4}$ & $\mathbf{5}$ \\
\hline 10 & -.2 & & 10.06 & 30.92 & 40.51 & 46.27 & 50.19 \\
& -.6 & & 32.36 & 50.03 & 57.60 & 61.99 & 64.93 \\
& -1 & & 57.58 & 71.43 & 76.67 & 79.52 & 81.35 \\
20 & -.2 & & 11.92 & 32.52 & 41.95 & 47.59 & 51.43 \\
& -.6 & & 38.72 & 55.45 & 62.44 & 66.44 & 69.11 \\
& -1 & & 69.48 & 81.49 & 85.63 & 87.75 & 89.05 \\
30 & -.2 & & 12.58 & 33.09 & 42.47 & 48.07 & 51.88 \\
& -.6 & & 41.04 & 57.41 & 64.20 & 68.06 & 70.61 \\
& -1 & & 73.86 & 85.19 & 88.92 & 90.77 & 91.88 \\
\hline
\end{tabular}

MTTF, mean times to failure.

Table 5 The percentage relative gain in MTTF of a parallel system.

\begin{tabular}{cccccccc}
\hline & \multirow{n}{n}{} & $\lambda$ & $\alpha_{2}$ & \multicolumn{7}{c}{ Parallel System } \\
\cline { 4 - 8 } & & $\mathbf{1}$ & $\mathbf{2}$ & $\mathbf{3}$ & $\mathbf{4}$ & $\mathbf{5}$ \\
\hline 10 & -1 & & 29.29 & 13.90 & 7.06 & 3.03 & 0.30 \\
20 & -1 & & 33.98 & 17.28 & 9.90 & 5.56 & 2.63 \\
30 & -1 & & 35.59 & 18.44 & 10.87 & 6.42 & 3.42 \\
\hline
\end{tabular}

MTTF, mean times to failure.

\section{ACKNOWLEDGMENTS}

The authors are grateful to the editor and reviewers for their suggestions in improving this paper.

\section{REFERENCES}

1. H.A. David, Bull. Inst. Int. Stat. 45 (1973), 295-300.

2. M. Ahsanullah, Bull. Malays. Math. Sci. Soc. 32(2) (2009), 101-117.

3. I. Bairamov,S. Kotz, M. Bekci, J. Appl. Stat. 28 (2001), 521-536.

4. M.I. Beg, M. Ahsanullah, Stat. Methodol. 5 (2008), 1-20.

5. J. Scaria, N.U. Nair, J. Appl. Stat. Sci. 14(5)(2005), 251-262.

6. J. Scaria, N.U. Nair, Stat. Pap. 49 (2008), 109-119.

7. N.U. Nair, J. Scaria, Statistics. 44 (2010), 155-167.

8. H.A. David, H.N. Nagaraja, Order Statistics, John Wiley and Sons, New York, USA 2003.

9. G.A. McIntyre, Aust. J. Agric. Res. 3 (1952), 385-390.

10. J. Scaria, B. Thomas, J. Appl. Stat. Sci. 21(1) (2014), 63-76.

11. C.D. Lai, M. Xie, Stochastic Ageing and Dependence for Reliability, Springer Science+Business Media Inc, New York, NY, USA, 2005.

12. H.W. Block, M.L. Ting, Commun. Stat. Theory Methods. 10 (1981), 749-762.

13. H. Joe, Multivariate Models and Dependence Concepts, Chapman and Hall, London, England, 1997.

14. J.D. EsaryF. Proschan, Ann. Math. Stat. 43 (1972), 651-655.

15. E.L. Lehmann, Ann. Math. Stat. 37 (1966), 1137-1153.

16. S. Karlin, Total Positivity, Stanford University Press, Stanford, CA, USA, 1968.

17. J.D. Esary, F. Proschan, D.W. Walkup, Ann. Math. Stat. 38 (1967), 1466-1474.

18. R.C. Gupta, Commun. Stat. Theory Methods. 45 (2016), 2342-2353.

19. N.L. Johnson, S. Kotz, Distributions in Statistics, Continuous Multivariate Distributions, John Wiley and Sons, New York and London, 1972.

20. J.Scaria,N.U. Nair, Biom. J. 41 (1999), 15-16.

21. S. Cambanis, J. Multivar. Anal. 7 (1977), 551-559.

22. P.W. Holland, Y.J. Wang, Commun. Stat. Theory Methods. 16 (1987), 193-206.

23. P.W. Holland, Y.J. Wang, Commun. Stat. Theory Methods. 16 (1987), 863-876.

24. R.E. Barlow, F. Proschan, Statistical Theory of Reliability and Life Testing, Silver Spring, USA, MD, 1981.

25. R.D. Gupta, D. Kundu, Aust. N. Z. J. Stat. 41 (1999), 173-188. 